\title{
Algebraic Kernel Method for Solving Fredholm Integral Equations
}

\author{
Mohammad Shami Hasso ${ }^{1,}$ a \\ ${ }^{1}$ Department of Mathematics, Faculty of Science and Health, \\ University of Koya, Koya, Erbil, Iraq \\ aemail: mohammad.shami@koyauniversity.org
}

\begin{abstract}
Keywords: Fredholm integral equations, Methods for solving linear Fredholm integral equations
\end{abstract}
\begin{abstract}
In this paper, we study the exact solution of linear Fredholm integral equations using some classical methods including degenerate kernel method and Fredholm determinants method. We propose an analytical method for solving such integral equations. This work has some goals related to suggested technique for solving Fredholm integral equations. The primary goal gives analytical solutions of such equations with minimum steps. Another goal is to compare the suggested method used in this study with classical methods. The final goal is that the propose method is an explicit formula that can be studied in detail for non-algebraic function kernels by using Taylor series expansion and for system of Fredholm integral equations.
\end{abstract}

\section{Introduction}

A variety of analytical and numerical methods are used to handle linear Fredholm integral equations such as successive approximation method, adomain decomposition method, modified decomposition method, direct computation method, Laplace transform method, Chebyshev collection method, the Taylor series method, Galerkin method, variational iteration method, spline collection method and other methods $[4,10]$.

Avazzadeh [2] , Bakodah [3] and Wazwaz [9] studied in particular a comparison between certain analytical and numerical methods for solving integral equations. Integral equations are one of the most useful mathematical tools in both pure and applied analysis [8]. The solutions of integral equations have a major role in the fields of science and engineering. The development of science has led to the formation of many physical laws, which, when restated in mathematical form, often appear as differential equations, an integral equations or an integro-differential equations or a system of these. Engineering problems can be mathematically described by differential equations or integral equations, and thus it play very important roles in the solution of practical problems. For example, Newton's law, stating that the rate of change of the momentum of a particle is equal to the force acting on it, can be translated into mathematical language as a differential equation. Similarly, problems arising in electric circuits, chemical kinetics, and transfer of heat in a medium can all be represented mathematically as differential equations or integral equations [6,7].

Fredholm integral equations arise in many scientific applications and can be derived from boundary value problems. Erik Ivar Fredholm (1866-1927) is the best remembered for his work on integral equations and spectral theory. Fredholm was a Swedish mathematician who established the theory of integral equations and his 1903 paper in Acta Mathematica played a major role in the establishment of operator theory [1].

An integral equation is said to be a Fredholm integral equation if the integral containing the unknown function $y(x)$ is characterized by fixed limits of integration of the form:

$$
y(x)=f(x)+\lambda \int_{a}^{b} K(x, t) y(t) d t,
$$

where $a$ and $b$ are constants, $y(x)$ is the unknown function to be determined, the function $f(x)$ and the kernel $K(x, t)$ are given real-valued functions and $\lambda$ is a parameter; see more details in $[1,6,8]$. 


\section{Methods}

There are a variety of methods to solve integral equations analytically and numerically. We solve linear Fredholm integral equations using analytical methods. Some of these methods are always known and used such as degenerate kernel method and Fredholm determinants method. These two methods were selected because they depend on the fixed laws can be applied and find the exact solution of any Fredholm integral equation.

\subsection{Degenerate Kernel Method}

The kernel $k(x, t)$ of Fredholm integral equation of the second kind is called degenerate if it is the sum of finite number of products of functions of $x$ alone by functions of $t$ alone; i.e., if it is of the form:

$$
k(x, t)=\sum_{k=1}^{n} a_{k}(x) b_{k}(t)
$$

We will consider the functions $a_{k}(x)$ and $b_{k}(t)$ for $k=1,2, \ldots, n$, continuous in the basic square $a \leq x, t \leq b$ are linearly independent. The integral equation with degenerate kernel (1)

$$
y(x)-\lambda \int_{a}^{b}\left[\sum_{k=1}^{n} a_{k}(x) b_{k}(t)\right] y(t) d t=f(x),
$$

is solved in the following manner.

Rewrite the equation (2) as:

$$
y(x)=f(x)+\lambda \sum_{k=1}^{n} a_{k}(x) \int_{a}^{b} b_{k}(t) y(t) d t,
$$

and introduce the notations:

$$
\int_{a}^{b} \sum_{k=1}^{n} b_{k}(t) y(t) d t=C_{k} \quad(k=1,2, \ldots, n) .
$$

Then the equation (3) becomes

$$
y(x)=f(x)+\lambda \sum_{k=1}^{n} C_{k} a_{k}(x)
$$

where $C_{k}$ for $k=1,2, \ldots, n$ are unknown constants, since the function $y(x)$ is unknown. Thus, the solution of an integral equation with degenerate kernel reduces to finding the constants $C_{k}$ $(k=1,2, \ldots, n)$. Putting the expression (5) into the integral equation (2), the equation takes the following form after simple manipulations:

$$
\sum_{m=1}^{n}\left[C_{m}-\int_{a}^{b} b_{m}(t)\left\{f(t)+\lambda \sum_{k=1}^{n} C_{k} a_{k}(t)\right\} d t\right] a_{m}(x)=0,
$$

Whence it follows, by virtue of the linear independence of the functions $a_{m}(x)$, that

$$
\left[C_{m}-\lambda \sum_{k=1}^{n} C_{k} \int_{a}^{b} a_{k}(t) b_{m}(t) d t\right]=\int_{a}^{b} b_{m}(t) f(t) d t \quad(m=1,2, \ldots, n)
$$

For the sake of brevity, we introduce the notations

$$
a_{k m}=\int_{a}^{b} a_{k}(t) b_{m}(t) d t \quad, \quad f_{m}=\int_{a}^{b} b_{m}(t) f(t) d t
$$


and find that $\quad C_{m}-\lambda \sum_{k=1}^{n} a_{k m} C_{k}=f_{m} \quad, \quad m=1,2, \cdots, n$

or, in expanded form:

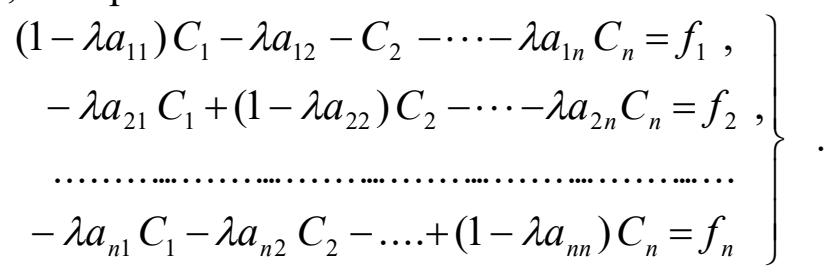

Using Cramer's rule or substituting method to solve the system (6) for finding the unknowns coefficients $C_{k}$.

The solution of the integral equation (2) is the function $y(x)$ defined by the equality : $y(x)=f(x)+\lambda \sum_{k=1}^{n} C_{k} a_{k}(x)$.

Example : Consider the integral equation $y(x)=x+\lambda \int_{0}^{1}\left(x t^{2}+x^{2} t\right) y(t) d t$, and using degenerate kernel method, we rewrite the given integral equation as $y(x)=x+\lambda x \int_{0}^{1} t^{2} y(t) d t+\lambda x^{2} \int_{0}^{1} t y(t) d t$ let $\quad c_{1}=\int_{0}^{1} t^{2} y(t) d t, c_{2}=\int_{0}^{1} t y(t) d t$, then

$$
y(x)=x+\lambda x c_{1}+\lambda x^{2} c_{2},
$$

$$
\begin{aligned}
& c_{1}=\int_{0}^{1} t^{2}\left[t+\lambda t c_{1}+\lambda t^{2} c_{2}\right] y(t) d t=\frac{1}{4}+\frac{\lambda c_{1}}{4}+\frac{\lambda c_{2}}{5} \\
& \Rightarrow \quad(20-5 \lambda) c_{1}-4 \lambda c_{2}=5
\end{aligned}
$$

and $\quad c_{2}=\int_{0}^{1} t\left[t+\lambda t c_{1}+\lambda t^{2} c_{2}\right] y(t) d t=\frac{1}{3}+\frac{\lambda c_{1}}{3}+\frac{\lambda c_{2}}{4}$,

$$
\Rightarrow \quad-4 \lambda c_{1}+(12-3 \lambda) c_{2}=5 \text {. }
$$

Solving (8) and (9) for $c_{1}$ and $c_{2}$, analytically we obtain

$$
c_{1}=\frac{60+\lambda}{240-120 \lambda-\lambda^{2}} \quad, \quad c_{2}=\frac{80}{240-120 \lambda-\lambda^{2}}
$$

Substituting these values of $c_{1}$ and $c_{2}$ into equation (7), we obtain the solution of the given integral equation as follows:

$$
y(x)=x+\frac{\lambda x(60+\lambda)}{240-120 \lambda-\lambda^{2}}+\frac{80 \lambda x^{2}}{240-120 \lambda-\lambda^{2}}
$$

as a result $y(x)=\frac{(240-60 \lambda) x+80 \lambda x^{2}}{240-120 \lambda-\lambda^{2}} \quad$ is a solution of the given Fredholm integral equation. 


\subsection{Fredholm Determinants Method}

The solution of the Fredholm integral equation of the second kind

$$
y(x)-\lambda \int_{a}^{b} K(x, t) y(t) d t=f(x)
$$

is given by the following formula

$$
y(x)=f(x)+\lambda \int_{a}^{b} R(x, t ; \lambda) f(t) d t,
$$

where the function $R(x, t ; \lambda)$ is called the Fredholm resolvent kernel of equation (10) and is defined by the equation

$$
R(x, t ; \lambda)=\frac{D(x, t ; \lambda)}{D(\lambda)},
$$

Provided that $D(\lambda) \neq 0$. Here $D(x, t ; \lambda)$ and $D(\lambda)$ are power series in $\lambda$ :

$$
\begin{aligned}
& D(x, t ; \lambda)=k(x, t)+\sum_{n=1}^{\infty} \frac{(-1)^{n}}{n !} B_{n}(x, t) \lambda^{n}, \\
& D(\lambda)=1+\sum_{n=1}^{\infty} \frac{(-1)^{n}}{n !} C_{n} \lambda^{n} .
\end{aligned}
$$

Whose coefficients are given by the following equations:

$$
\begin{aligned}
& B_{n}(x, t)=C_{n} k(x, t)-n \int_{a}^{b} k(x, z) B_{n-1}(z, t) d z, \\
& C_{n}=\int_{a}^{b} B_{n-1}(z, z) d z .
\end{aligned}
$$

Knowing that the coefficients $C_{0}=1$ and $B_{0}(x, t)=k(x, t)$.

We can use the formulas (15) and (16) to calculate $C_{n}$ and $B_{n}(x, t)$ for $n=1,2,3, \cdots$ analytically, [5].

Example: We apply Fredholm Determinants method (Recurrence Relations) to solve the integral equation

$$
y(x)=x+\lambda \int_{0}^{1}\left(x t^{2}+x^{2} t\right) y(t) d t .
$$

As we note that

$$
\begin{aligned}
& B_{0}(x, t)=k(x, t)=x t^{2}+x^{2} t, \quad C_{0}=1, \\
& C_{1}=\int_{0}^{1} B_{0}(z, z) d z=\int_{0}^{1} 2 z^{3} d z=\frac{1}{2}, \\
& B_{1}(x, t)=C_{1} k(x, t)-\int_{a}^{b} k(x, z) B_{0}(z, t) d z=\frac{1}{2}\left(x t^{2}+x^{2} t\right)-\int_{0}^{1}\left(x z^{2}+x^{2} z\right)\left(z t^{2}+z^{2} t\right) d z
\end{aligned}
$$

Hence

$$
B_{1}(x, t)=x t\left(\frac{x+t}{4}-\frac{x t}{3}+\frac{1}{5}\right) .
$$

and

$$
C_{2}=\int_{0}^{1} B_{1}(z, z) d z=\int_{0}^{1}\left[z^{2}\left(\frac{z}{2}-\frac{z^{3}}{3}-\frac{1}{5}\right)\right] d z=-\frac{1}{120},
$$


$B_{2}(x, t)=C_{2} k(x, t)-\int_{a}^{b} k(x, z) B_{1}(z, t) d z=\frac{-1}{120}\left(x t^{2}+x^{2} t\right)-\int_{0}^{1}\left(x z^{2}+x^{2} z\right)\left[\left(z t\left(\frac{z+t}{4}-\frac{z t}{3}-\frac{1}{5}\right)\right] d z=0\right.$.

As a result $B_{2}(x, t)=0$ therefore $B_{n}(x, t)=0$ and $C_{n}=0$ for all $n \geq 3$. Substituting these values into (15) and (16), we have

$D(x, t ; \lambda)=k(x, t)+\sum_{n=1}^{\infty} \frac{(-1)^{n}}{n !} B_{n}(x, t) \lambda^{n}=x t^{2}+x^{2} t-x t \lambda\left(\frac{x+t}{4}-\frac{x t}{3}-\frac{1}{5}\right)$,

and $\quad D(\lambda)=1+\sum_{n=1}^{\infty} \frac{(-1)^{n}}{n !} C_{n} \lambda^{n}=1-\frac{\lambda}{2}-\frac{\lambda^{2}}{120}$,

we get

$$
R(x, t ; \lambda)=\frac{D(x, t ; \lambda)}{D(\lambda)}=\frac{x t^{2}+x^{2} t-x t \lambda\left(\frac{x+t}{4}-\frac{x t}{3}-\frac{1}{5}\right)}{1-\frac{\lambda}{2}-\frac{\lambda^{2}}{120}}
$$

Hence, the exact solution of the given integral equation is

$$
\begin{aligned}
y(x) & =x+\int_{0}^{1} R(x, t ; \lambda) f(t) d t \\
& =x+\int_{0}^{1}\left[\frac{x t^{2}+x^{2} t-x t \lambda\left(\frac{x+t}{4}-\frac{x t}{3}-\frac{1}{5}\right)}{1-\frac{\lambda}{2}-\frac{\lambda^{2}}{120}} t\right] d t \\
& =\frac{(240-60 \lambda) x+80 \lambda x^{2}}{240-120 \lambda-\lambda^{2}} .
\end{aligned}
$$

\section{Results and Discussions}

Solving linear Fredholm integral equations analytically are sometimes very difficult, and they are required more computational steps. In order to have the solutions for such problems, we propose an analytical technique for solving Fredholm integral equations. The suggested method is named by algebraic function kernel method. The method depends on the kernel of linear Fredholm integral equations. Therefore, we have two main cases based on the kernels for such equations:

Case One . If the kernel of the integral equation is algebraic function of degree $n$, then the solution of the integral equation with algebraic function kernel is also algebraic function of degree $n$, where $n=1,2,3, \ldots$

We consider the non-homogenous linear Fredholm integral equation of the second kind

$$
y(x)=f(x)+\lambda \int_{0}^{1} K(x, t) y(t) d t .
$$

In this case, the variable $t$ appear with the variable $x$ in the kernel of the integral equation, i.e. $K(x, t)=x^{n} t^{m}+x^{n-1} t^{m-1}+\cdots+t^{r} \quad, \quad n, m, r=1,2,3, \cdots$.

and the integral equation (17) becomes

$$
y(x)=f(x)+\lambda \int_{0}^{1}\left(x^{n} t^{m}+x^{n-1} t^{m-1}+\cdots+t^{r}\right) y(t) d t \quad, \quad n, m, r=1,2,3, \cdots .
$$


Then the solution of the integral equation is

$$
y(x)=a x^{n}+a_{1} x^{n-1}+\cdots+a_{n} .
$$

Substituting equation (19) into equation (18), we have

$$
a x^{n}+a_{1} x^{n-1}+\cdots+a_{n}=f(x)+\lambda \int_{0}^{1}\left(x^{n} t^{m}+x^{n-1} t^{m-1}+\cdots+t^{r}\right)\left(a t^{n}+a_{1} t^{n-1}+\cdots+a_{n}\right) d t,
$$

Now we solve the second term in right side of equation (20) to get

$$
\begin{aligned}
a x^{n}+a_{1} x^{n-1}+\cdots & +a_{n}=f(x)+\lambda\left[\left(\frac{a}{n+m+1}+\frac{a_{1}}{n+m}+\cdots+\frac{a_{n}}{m+1}\right) x^{n}+\right. \\
& \left.+\left(\frac{a}{n+m}+\frac{a_{1}}{n+m-1}+\cdots+\frac{a_{n}}{m}\right) x^{n-1}+\left(\frac{a}{n+r+1}+\frac{a_{1}}{n+r}+\cdots+\frac{a_{n}}{r+1}\right)\right]
\end{aligned}
$$

Both sides of the equation (21) are equal only when the coefficients of the variable $x$ are equally in both sides, meaning that

$$
\begin{aligned}
& a=\lambda\left(\frac{a}{n+m+1}+\frac{a_{1}}{n+m}+\frac{a_{n}}{m+1}\right)
\end{aligned}
$$

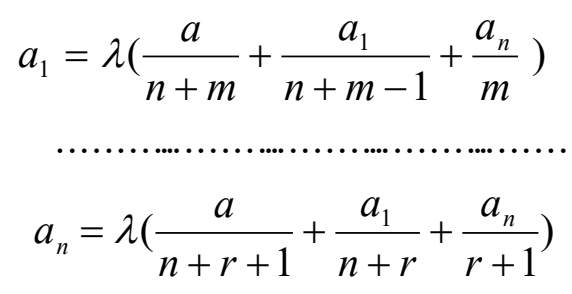

We write the matrix $A$ of eigen values to the parameter $\lambda$ as follows:

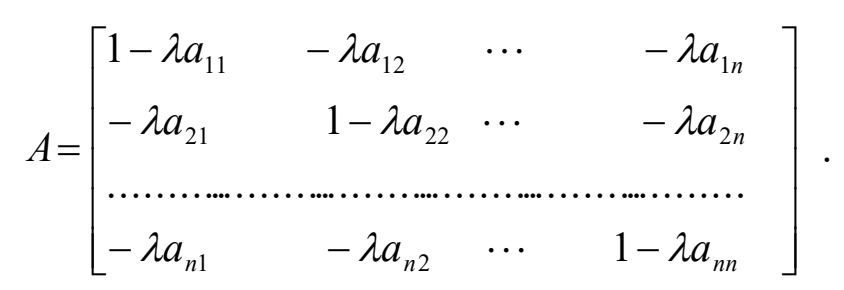

From equation (22) the determinant of the matrix $A$ is

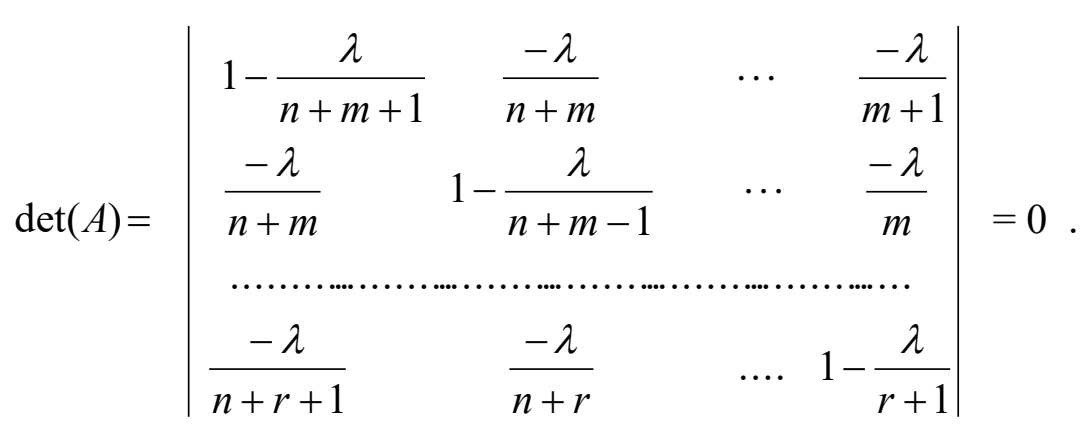

After known $n, m, r$ and substituting into equation (22) or (23) to find the values of the coefficients of the variable $x$ by substituting method or Cramer's rule, then substitute the values of the coefficients of the variable $x$ into equation (19) to find the solution of the integral equation (18). 
Example: To solve the integral equation

$y(x)=x+\lambda \int_{0}^{1}\left(x t^{2}+x^{2} t\right) y(t) d t$

by algebraic function kernel method, we observe the kernel of the integral equation $K(x, t)=x t^{2}+x^{2} t$

is an algebraic function of second degree then the solution of given integral equation is also an algebraic function of second degree i.e.

$$
y(x)=a x^{2}+b x+c
$$

then substituting the given algebraic equation into the given integral equation to obtain

$$
\begin{aligned}
a x^{2}+b x+c & =x+\lambda \int_{0}^{1}\left(x t^{2}+x^{2} t\right)\left(a t^{2}+b t+c\right) d t \\
& =x+\lambda \int_{0}^{1}\left(a x t^{4}+b x t^{3}+c x t^{2}+a x^{2} t^{3}+b x^{2} t^{2}+c x^{2} t\right) d t \\
& =x+\frac{a \lambda x}{5}+\frac{b \lambda x}{4}+\frac{c \lambda x}{3}+\frac{a \lambda x^{2}}{4}+\frac{b \lambda x^{2}}{3}+\frac{c \lambda x^{2}}{2} \\
& =\left(1+\frac{a \lambda}{5}+\frac{b \lambda}{4}+\frac{c \lambda}{3}\right) x+\left(\frac{a \lambda}{4}+\frac{b \lambda}{3}+\frac{c \lambda}{2}\right) x^{2}
\end{aligned}
$$

By comparing the coefficients of the same power of $x$, then we can find $a, b$ and $c$ as follows

$$
\begin{aligned}
& a=\frac{a \lambda}{4}+\frac{b \lambda}{3}+\frac{c \lambda}{2}, \\
& b=1+\frac{a \lambda}{5}+\frac{b \lambda}{4}+\frac{c \lambda}{3}
\end{aligned}
$$

and $c=0$

Hence

$$
\begin{gathered}
\left(1-\frac{\lambda}{4}\right) a-\frac{\lambda}{3} b=0 \\
-\frac{\lambda}{5} a+\left(1-\frac{\lambda}{4}\right) b=1
\end{gathered}
$$

From equations (25) and (26) we have the matrix

$$
A=\left[\begin{array}{cc}
1-\frac{\lambda}{4} & \frac{-\lambda}{3} \\
\frac{-\lambda}{5} & 1-\frac{\lambda}{4}
\end{array}\right]=\left[\begin{array}{l}
0 \\
1
\end{array}\right] \quad, \quad \operatorname{det}(A)=\frac{240-120 \lambda-\lambda^{2}}{240}
$$


By Cramer's rule we obtain

$$
a=\frac{\left|\begin{array}{cc}
0 & \frac{-\lambda}{3} \\
1 & 1-\frac{\lambda}{4}
\end{array}\right|}{\frac{240-120 \lambda-\lambda^{2}}{240}}=\frac{80 \lambda}{240-120 \lambda-\lambda^{2}}
$$

and

$$
b=\frac{\left|\begin{array}{cc}
1-\frac{\lambda}{4} & 0 \\
\frac{-\lambda}{5} & 1
\end{array}\right|}{\frac{240-120 \lambda-\lambda^{2}}{240}}=\frac{240-60 \lambda}{240-120 \lambda-\lambda^{2}}
$$

Now substituting (27) and (28) and $c=0$ into (24), we obtain

$$
\begin{aligned}
y(x) & =\frac{80 \lambda x^{2}}{240-120 \lambda-\lambda^{2}}+\frac{(240-60 \lambda) x}{240-120 \lambda-\lambda^{2}}+0 \\
& =\frac{(240-60 \lambda) x+80 \lambda x^{2}}{240-120 \lambda-\lambda^{2}} .
\end{aligned}
$$

Which is the solution of the given Fredholm integral equation.

Case Two. If the kernel of the integral equation is non-algebraic function, then we use Taylor series expansion method in order to transform non- algebraic kernel to algebraic one. After that we use the same process as we used in case one for having some analytical approximate solutions for such integral equations.

Generally speaking, the proposed method gives a good step forward regarding analytical solutions for solving linear Fredholm integral equations with algebraic and non-algebraic kernel functions. It provides a simple mathematical procedure to solve problems in integral equations analytically. On the other hand, there are some difficulties in applying the suggested method to linear Fredholm integral equations. One difficulty is that having algebraic kernel functions in higher order gives a system of equations, and this is sometimes computationally very expensive. Another difficulty is related to non - algebraic kernel functions because some non - algebraic kernel functions cannot be transformed to the algebraic kernel function by using Taylor series expansion method.

\section{Conclusion}

Solving Fredholm integral equations analytically are sometimes a difficult task in mathematics. This difficulty generally depends on the variety of the kernel functions of such equations. In this paper, an analytical method is proposed for solving Fredholm integral equations when the kernel and absolute terms are algebraic functions, i.e. the algebraic function kernel method depends on the kernel of linear Fredholm integral equations. Therefore, if the kernel of the integral equation is algebraic function of degree $n$, then the solution of the integral equation with algebraic function kernel is also algebraic function of degree $n$, where $n=1,2,3, \ldots$. It can be argued that the proposed 
method in this work is more applicable and computationally cheap for solving Fredholm integral equations with algebraic kernels, because it has less steps compared to the other methods as we mentioned in literature review. The suggested method can also be applied for non-algebraic kernel functions. Interestingly, we can use the ideal of Taylor series expansion for such kernels and then apply our proposed technique. The proposed technique can also be applied for solving linear system of Fredholm integral equations.

\section{References}

[1] Abdul J. jerri, "Introduction to Integral Equation with applications" Marcel Dekker, Inc ,New York, 1985.

[2] Avazzadeh Z., Heydari M. and Loghmani G.B., A comparison between solving two dimentional integral equations by the traditional collection method and radial basis functions, Applied mathematical sciences, Vol. 5, No.23, pp: 1145-1152, 2011.

[3] Bakodah H.O., A comparison study between a Chebyshev collection method and the Adomian decomposition method for solving linear system of Fredholm integral equations of the second kind, JKAU:Sci., Vol. 24 No. 1, pp: 49-59, 2012.

[4] Hameed H. Hameed, Hayder M. Abbas and Zahraa A. Mohammed, The Taylor series method for solving linear Fredholm integral equations of the second kind using MATLAB, Journal of Babylon University, Pure and applied Sciences, No. 1, Vol. 19, 2011.

[5] Krasnov M., Kiselev A., Problems and Exercises in Integral Equations, Mir publishers, Moscow, 1971.

[6] Rahman M., " Integral Equation and their applications", Wit Press Southampton, Boston, 2007.

[7] Shidfar A. and Molabahrami A., Solving system of integral equations by an analytic method, mathematical and computer modeling journal, vol. 54, pp:28-35, 2011.

[8] Tricomi F.G., Integral Equation, Inter science Publisher, INC. New york,1957.

[9] Wazwaz Abdul-Majid, A comparison between the variational iteration method and Adomian decomposition method, Journal of computational and applied mathematics 207 , pp: 129-136, 2007.

[10] Wazwaz Abdul-Majid, Linear and Nonlinear Integral Equations Methods and Applications Higher Education press, Beijing and springer-verlag Berlin Heidelberg, 2011. 